\title{
Xantomatosis cerebrotendinosa. Reporte de un caso en el Perú: Evaluación clínica integral y estudios de neuroimágenes.
}

\author{
Cerebrotendinous Xanthomatosis. Report of a case in Peru: Comprehensive clinical evaluation and \\ neuroimaging studies.
}

Marco A. Castañeda ${ }^{1,2}$, César Durán Espinoza ${ }^{2,3}$, José Cabanillas Lapa ${ }^{2,4}$

\section{RESUMEN}

Se presenta el caso de una paciente de 73 años de edad que, a los 30 años aproximadamente, comenzó a quejarse de dolor al caminar, localizando la molestia a nivel de las regiones aquilianas, con subsecuente aumento de volumen; al paso del tiempo, estas molestias la obligaron a efectuar consulta médica. Los análisis de laboratorio mostraron severa dislipidemia mixta. Al lado de información de significativa declinación cognitiva, provista por familiares (vgr., (i.e., olvidos frecuentes, desorientación, atención disminuida, concentración pobre), hubo evidencia de ánimo fluctuante, labilidad emocional, crisis ansiosas evolucionando hacia ataques de pánico. El test minicognitivo de Folstein, mostró severo estado demencial y en el examen neurológico se constataron ataxia cerebelosa y signos de piramidalismo parcial. El examen oftalmológico puso en evidencia xantelasmas, cataratas y un denso arco senil. El estudio del cerebro con resonancia magnética (RM) mostró el daño encefálico y signos sugestivos de depósitos del colastenol en el SNC. La presencia de xantomas, los hallazgos oftalmológicos, la demencia definidamente progresiva y la ataxia cerebelosa fueron hallazgos clínicos que permitieron establecer el diagnóstico de xantomatosis cerebrotendinosa.

PALABRAS CLAVE : Xantomatosis cerebrotendinosa, demencia, atrofia cerebral.

\section{SUMMARY}

The case of a 73 years-old woman that, since approximately the age of 30 years started to complain of pain when walking, is presented. The symptom was mainly located in the acchillean regions which, as time advanced, showed gradual volume increase and, finally, forced her to seek medical evaluation. Accompanying relatives reported a several years' history of gradually increasing cognitive difficulties (i.e., forgetfulness, disorientation, poor attention and concentration), fluctuating mood (from periods of good humor switching to sudden episodes of sadness and crying spells), emotional lability and anxiety crises evolving into brief panic attacks. The Mini-cognitive Fenton Test confirmed severe dementia and the neurological evaluation showed cerebellar ataxia and partial pyramidalism. The ophthalmological examination revealed xanthelasmas, cataracts and dense arcus senilis. Xanthomas were detected in the Achillean tendons of both lower extremities. Auxiliary laboratory and densitometric tests demonstrated mixed dyslipidemia and dorsal-lumbar osteoporosis, respectively, and magnetic resonance imaging of the brain (RMC) confirmed SNC damage and suggested deposits of cholestenol, thus confirming the diagnosis of Cerebroitendinous Xanthomatosis.

KEYWORDS : Cerebrotendinous Xanthomatosis, dementia, brain atrophy.

Servicio de Neurología, Hospital Nacional Dos de Mayo. Lima, Perú.

Facultad de Medicina, Universidad Nacional Mayor de San Marcos. Lima, Perú.

Servicio de Oftalmología, Hospital Nacional Dos de Mayo. Lima, Perú.

Servicio de Patología, Hospital Nacional Dos de Mayo. Lima, Perú. 


\section{INTRODUCCIÓN}

La xantomatosis cerebrotendinosa es una enfermedad hereditaria autosómica recesiva producida por un déficit en la enzima mitocondrial esterol 27-hidroxilasa (CYP27A1) (1).

El gen CYP27A1 humano se localizó en el brazo del cromosoma 2q. Esta enzima participa en las síntesis de ácidos biliares. Su deficiencia produce una reducción de la síntesis de ácido quenodeoxicólico (AQDC ) y un aumento de su producto de oxidación el colastenol, que se deposita en diferentes tejidos especialmente en el cerebro , cristalino y los tendones, participa junto a otras hidroxilasas en la 25-hidroxilación de la vitamina D3 (2), con la deficiencia de ésta se desarrolla osteoporosis precoz.

Las manifestaciones clínicas se presentan en relación al depósito de colastenol en diferentes tejidos como tendones , cristalino y sistema nervioso central (3). Los pacientes inicialmente presentan diarrea durante la infancia y cataratas juveniles y , en etapas más avanzadas de la enfermedad aparecen xantomas tendinosos, piramidalismo, signos de compromiso cerebeloso, convulsiones, y menos frecuentemente ateroesclerosis precoz $(4,5)$ y senescencia prematura de la retina (6).

La expresión clínica de la enfermedad se expresa en trastorno del desarrollo psicomotor, demencia, ataxia, trastornos psiquiátricos, epilepsia y signos neurológicos focales como piramidalismo, signos de parkinsonismo y neuropatía periférica. Estos serían secundarios al depósito del material lipídico en los hemisferios cerebelosos, globus pallidus y pedúnculos cerebrales. En las imágenes (RM), además, es común observar atrofia cerebral. El diagnóstico final se realiza con la constatación de niveles elevados de colestanol plasmático, prueba que no se encuentra aún en nuestro país.

La investigación con RMN del encéfalo aportan información de apoyo para la sospecha diagnóstica. El estudio genético no es necesario en el proceso del diagnóstico.

Con la finalidad llamar la atención sobre sus características clínicas y radiológicas, presentamos un caso clínico de xantomatosis cerebro-tendinosa, basando el diagnóstico en los resultados histopatológicos.

\section{Descripción del caso}

Paciente de 73 años de edad natural del Departamento de Lambayeque, quien reside de hace varios años en Lima. Cursó la primaria, y se ha dedicado a las labores fundamentalmente de las tareas del hogar, mal informante debido a su deterioro intelectual. El motivo de la consulta inicial fue dolor a nivel de los tendones aquilianos en especial cuando estaba relacionado con caminatas prolongadas, así mismo, refirió dolor a nivel dorso - lumbar vertebral. De considerable importancia fueron las extremas alteraciones en la entrevista de la inconsistencia de datos, por imprecisiones en las fechas, dubitaciones marcadas de datos en su infancia, así como en su escolaridad, era marcada la indiferencia emocional frente a estos defectos. Fue necesaria la ayuda de uno de sus hijos que convivía con la paciente, quien informó que siempre la acompañaba en sus actividades de la vida diaria las consultas médicas por los disturbios de memoria y poder proporcionar datos fiables y correctos. Otro hijo varón, quien reside en Chile, presenta xantomas en codos y las muñecas. No pudo ser evaluado por no acceder a la petición médica. No hubieron antecedentes de importancia de factores de riesgo para enfermedad condicionante de compromiso vascular cerebral.

El examen clínico y por sistemas: mostró a una paciente con funciones vitales normales. En la facies destacaba presencia de arco blanquecino denso bilateral y de xantelasma en el párpado superior izquierdo, así como opacidad en ambos cristalinos, por exploración oftalmológica (Figura $1(\mathrm{~A}, \mathrm{~B}, \mathrm{C})$ ). La exploración de los pares craneales normales en su totalidad, que incluyó el fondo del ojo, sin alteraciones.

La exploración de la columna vertebral fue normal, no hubo dolor a la maniobra de Dandy, sin embargo ante la insistencia de la paciente, por molestia dolorosa se efectuó estudio radiográfico de la misma y además densitometría ósea en el que se constató severa osteoporosis dorso-lumbar (figura 2). Este hallazgo obligó a determinar el perfil lipídico en la paciente. Las cifras del colesterol total fue de CT: $356 \mathrm{mg} /$ dl y de triglicéridos: $175 \mathrm{mg} / \mathrm{dl}$, constituyéndose en dislipidemia mixta.

El hallazgo de la marcada osteopenia, obligó a determinar el perfil lipídico de la paciente determinándose dislipidemia mixta: CT:356 mg/dl y nivel de triglicéridos $175 \mathrm{mg} / \mathrm{dl}$, colesterol LDL: 268 $\mathrm{mg} / \mathrm{dl}$; hallazgos superiores a sus valores normales 


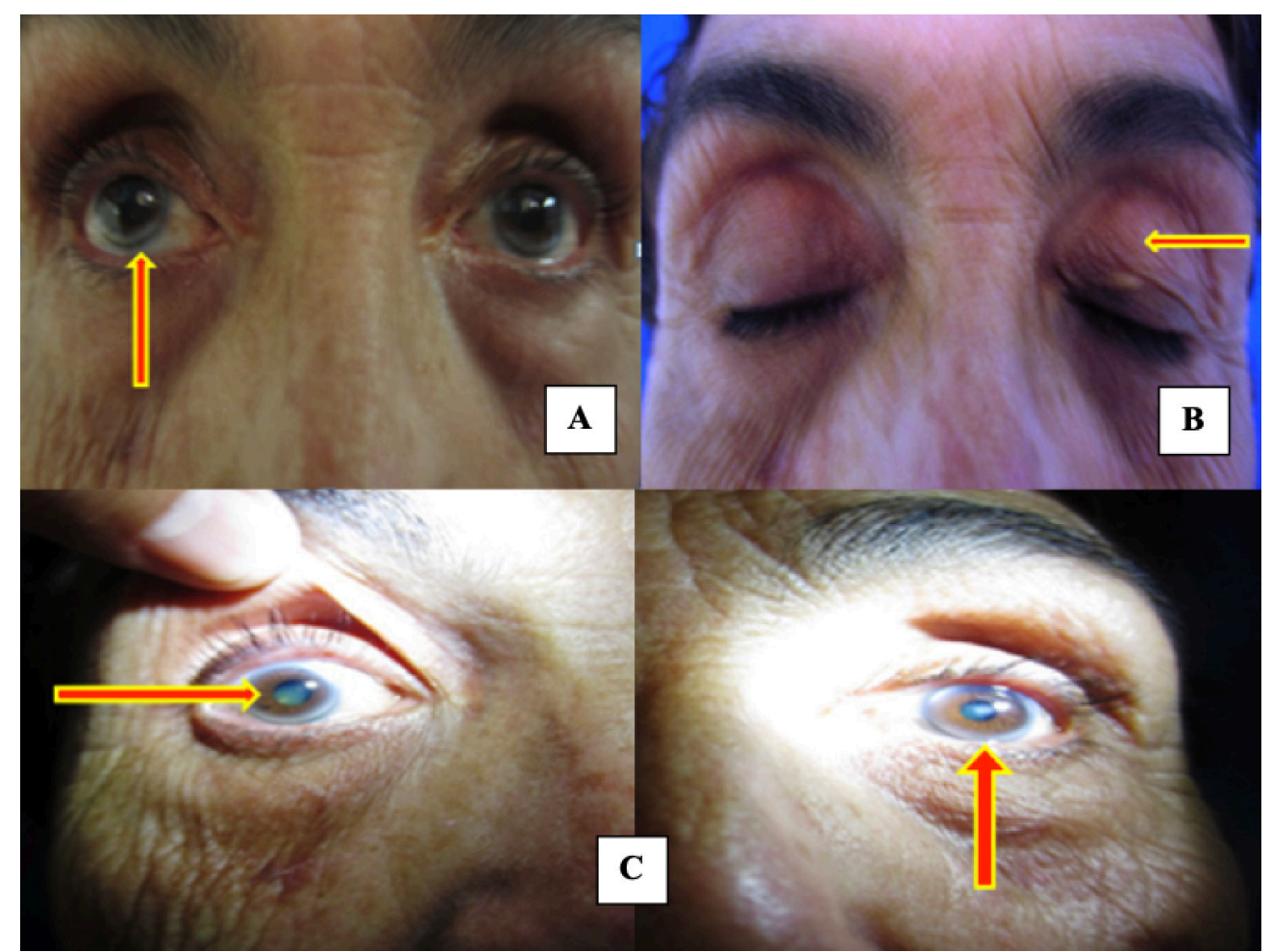

Figura 1. A: Arco senil denso; B: Xantelasma palpebral; C: Cataratas.

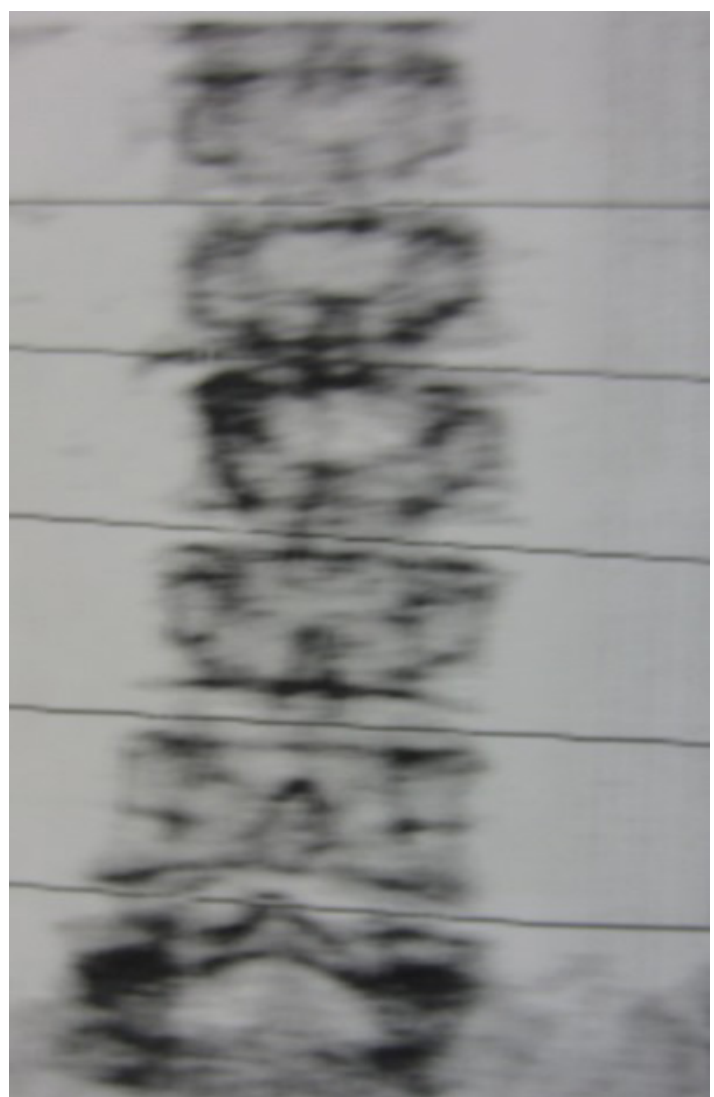

Figura 2. Severa osteoporosis dorso-lumbar. 


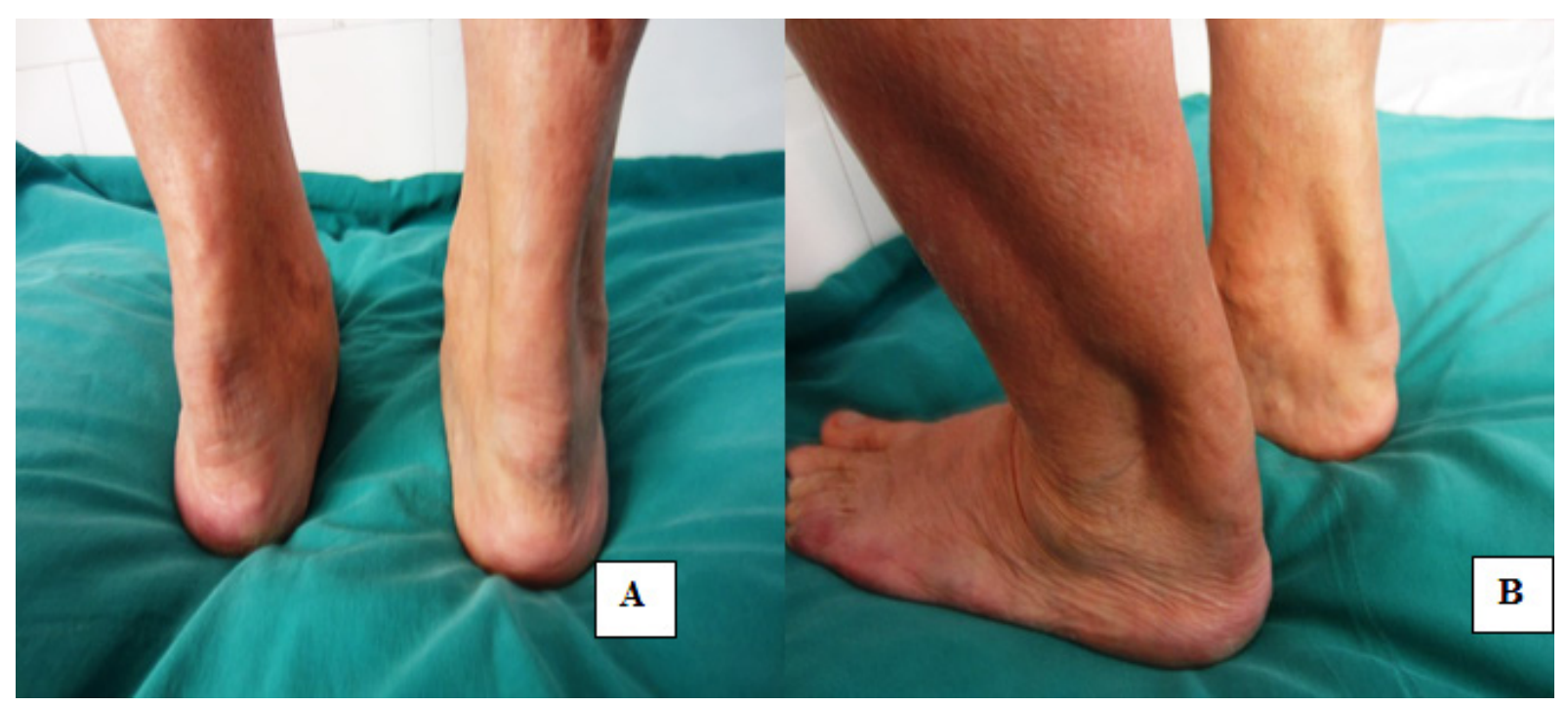

Figura 3. Presencia de xantomas en vista posterior (A) y vista lateral (B).

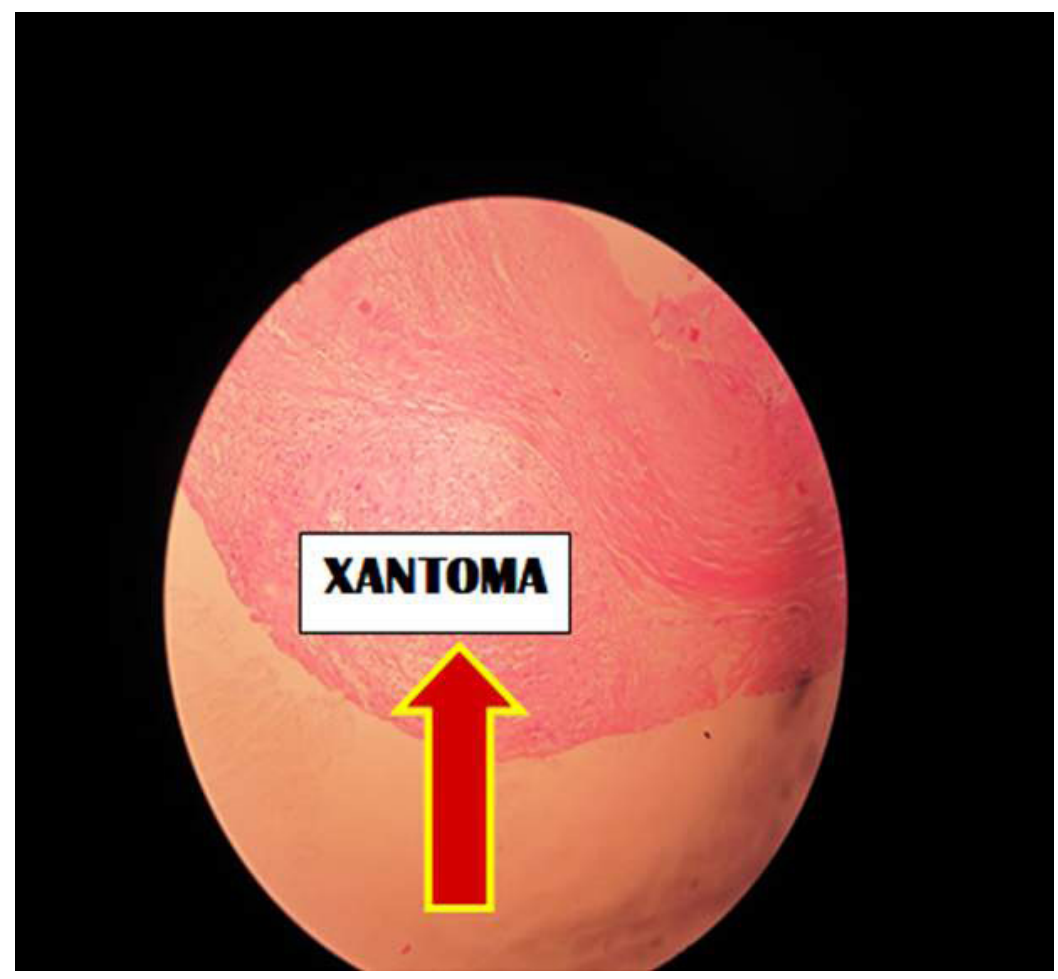

Figura 4. Area de proliferación histiocitaria (XANTOMA).

y frecuentes hallazgos en la XCT. Esta condición por la interferencia del colastenol elevado impide la formación de vitamina $\mathrm{D}$ y con ello la progresiva descalcificación, el resto de la exploración por sistemas al referirnos al corazón, pulmones, abdomen y aparato urogenital normales.

Al explorar los miembros inferiores, llamó poderosamente la atención el volumen de las regiones aquilianas aumentado y en forma de conos invertidos por la presencia de xantomas (figura 3 (A y B)). Se efectuó biopsia de la región aquiliana que mostró el tendón alterado con un área de proliferación histiocitaria en relación a lesión xantomatosa (figura 4), y la arquitectura del tendón reemplazada por tejido mixoide (figura 5).

La exploración neurológica mostró marcadas alteraciones en la esfera emocional, adoptando un comportamiento infantil, con variaciones en el ánimo 


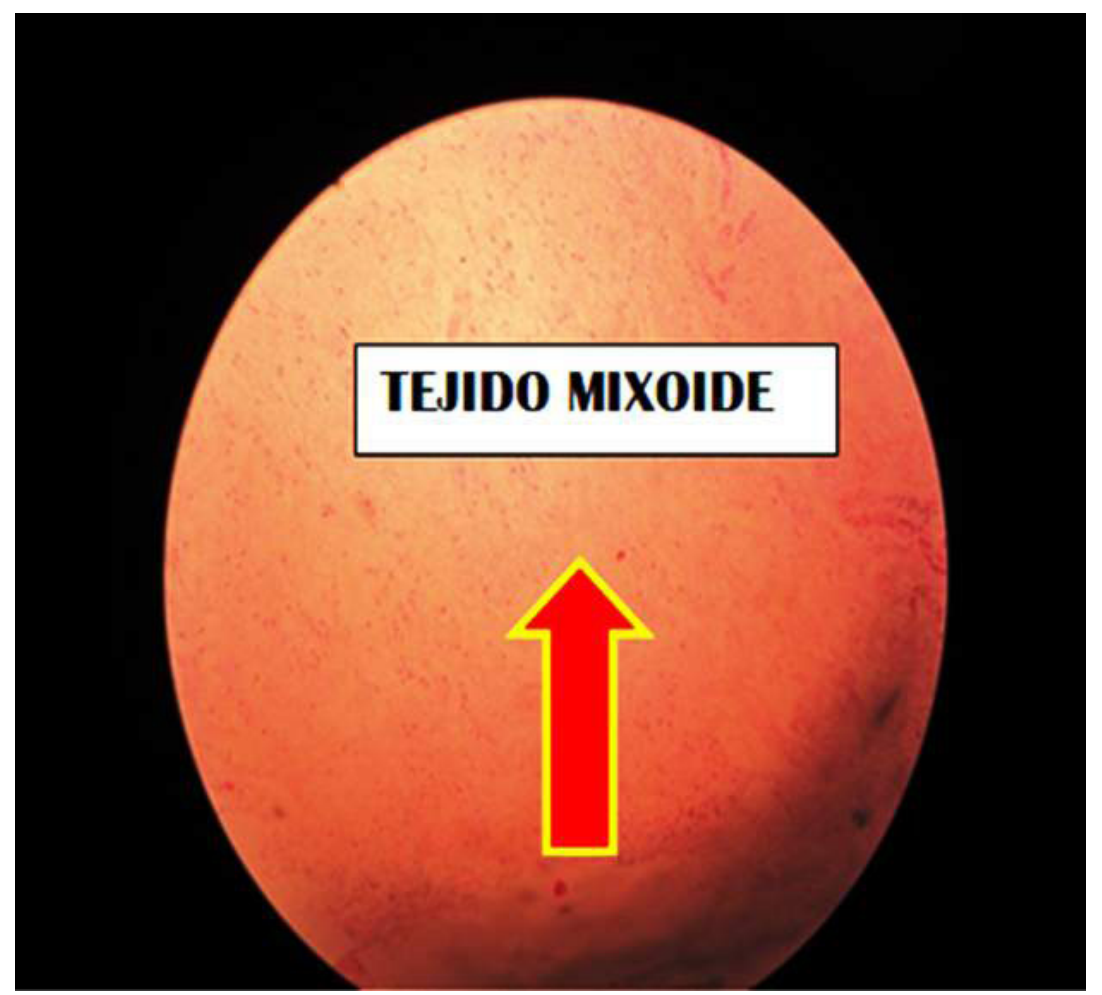

Figura 5. Arquitectura del tendón (tejido mixoide)

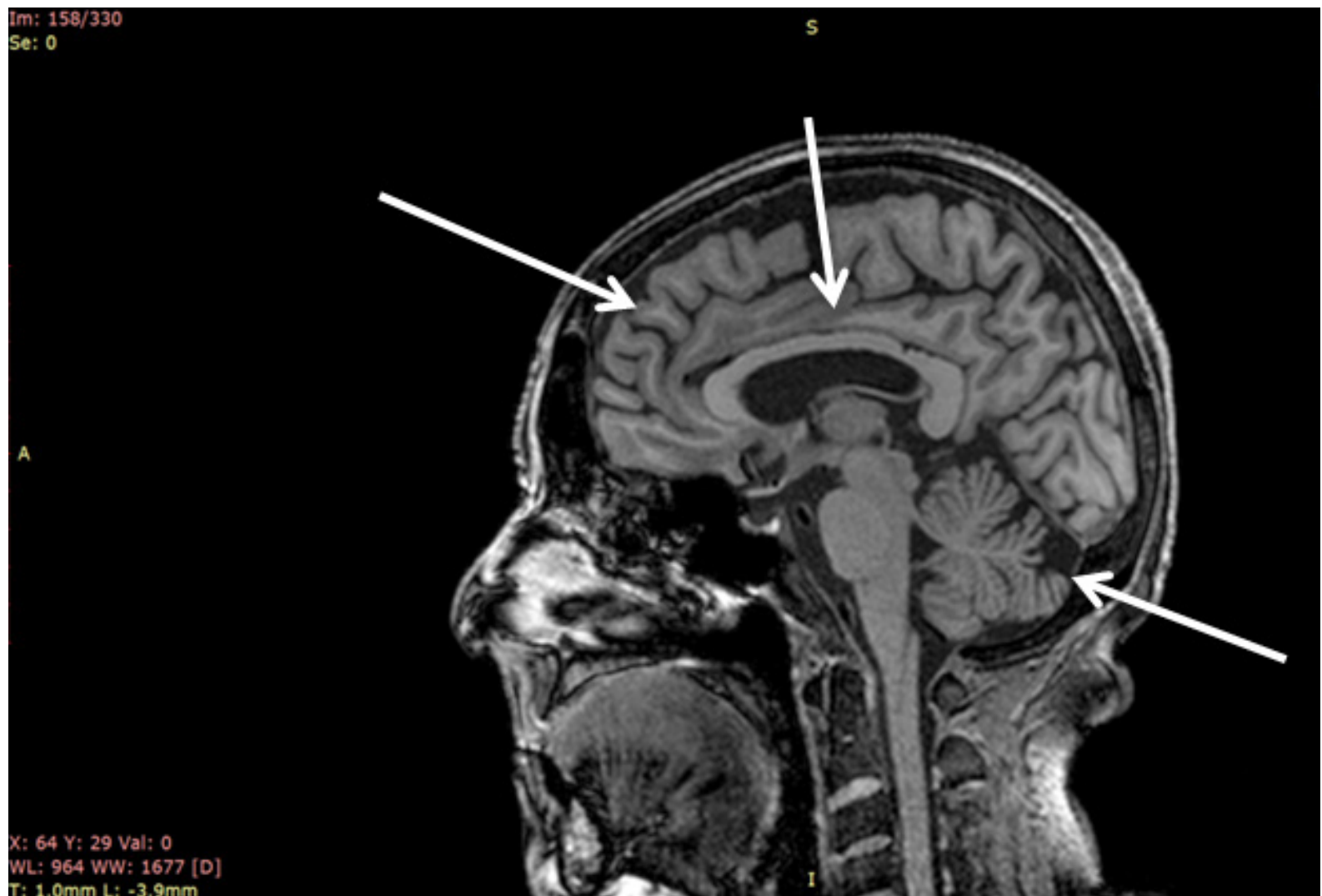

Figura 6. T1: atrofia fronto parieto temporal, del cuerpo calloso y el cerebelo. 


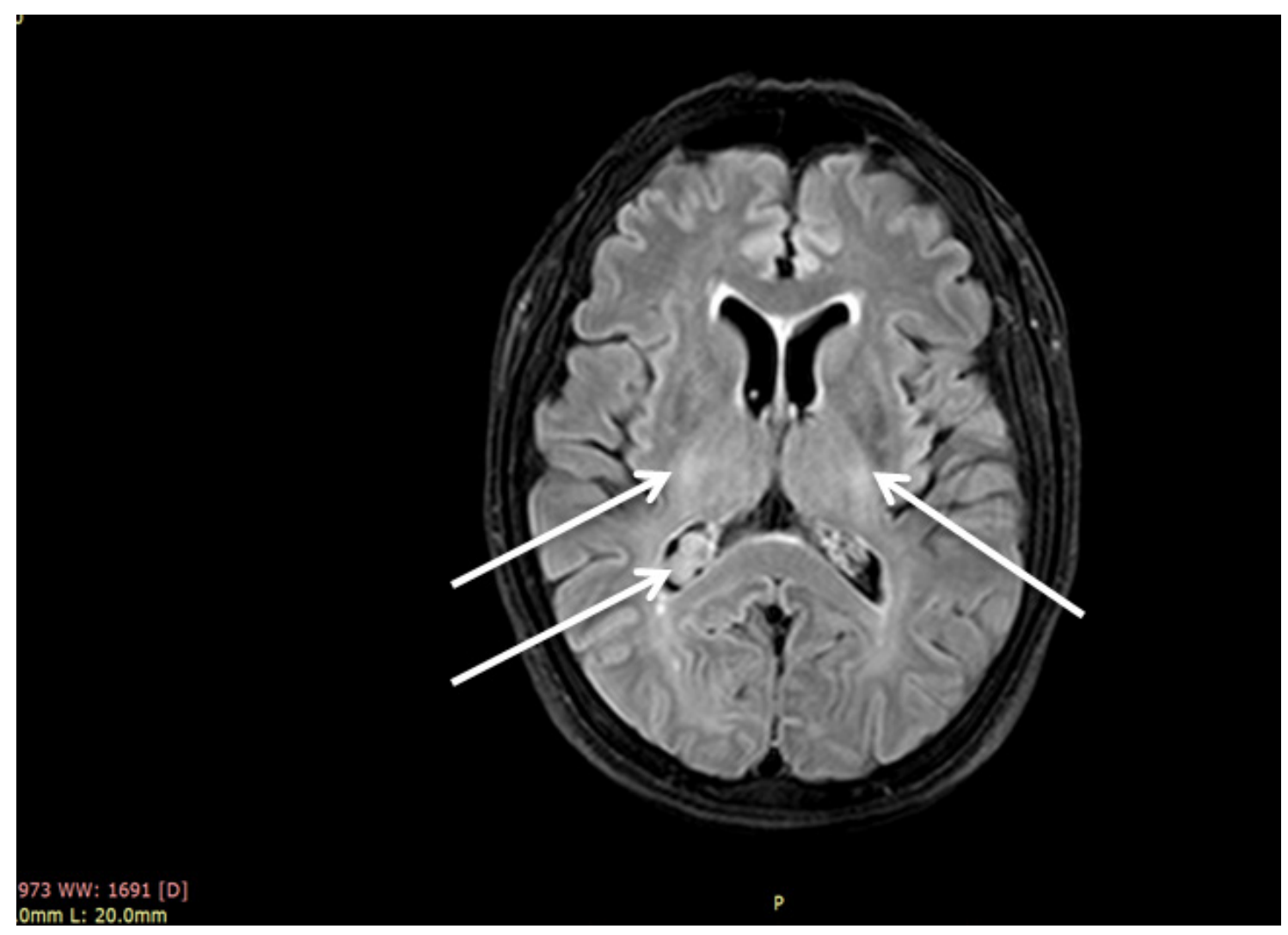

Figura 7. Señales de hiperintensidad en las capsulas internas y xantoma del plexo coroideo derecho .

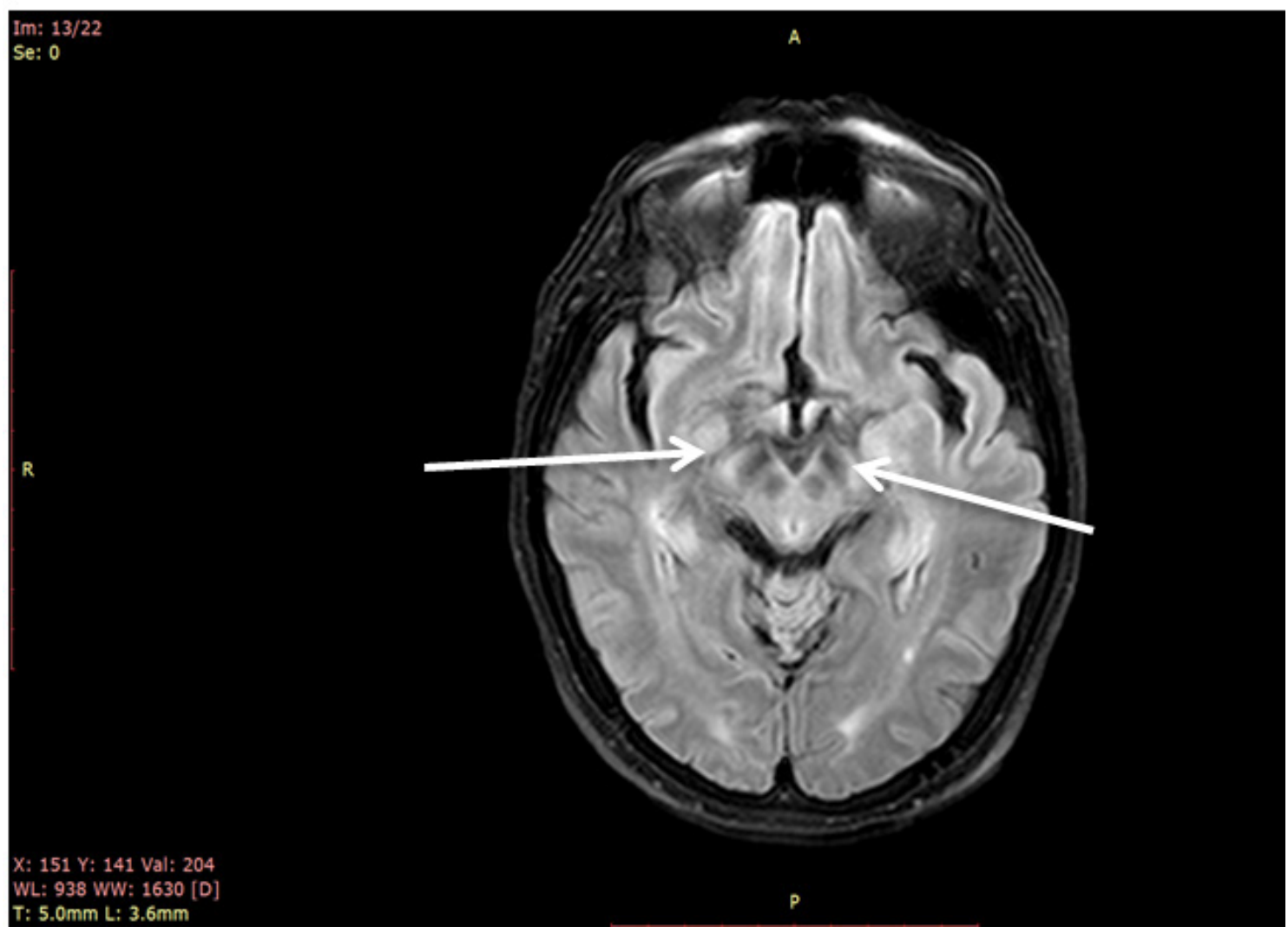

Figura 8. Señales de hiperintensidad en el pie de los pedunculos cerebrales. 


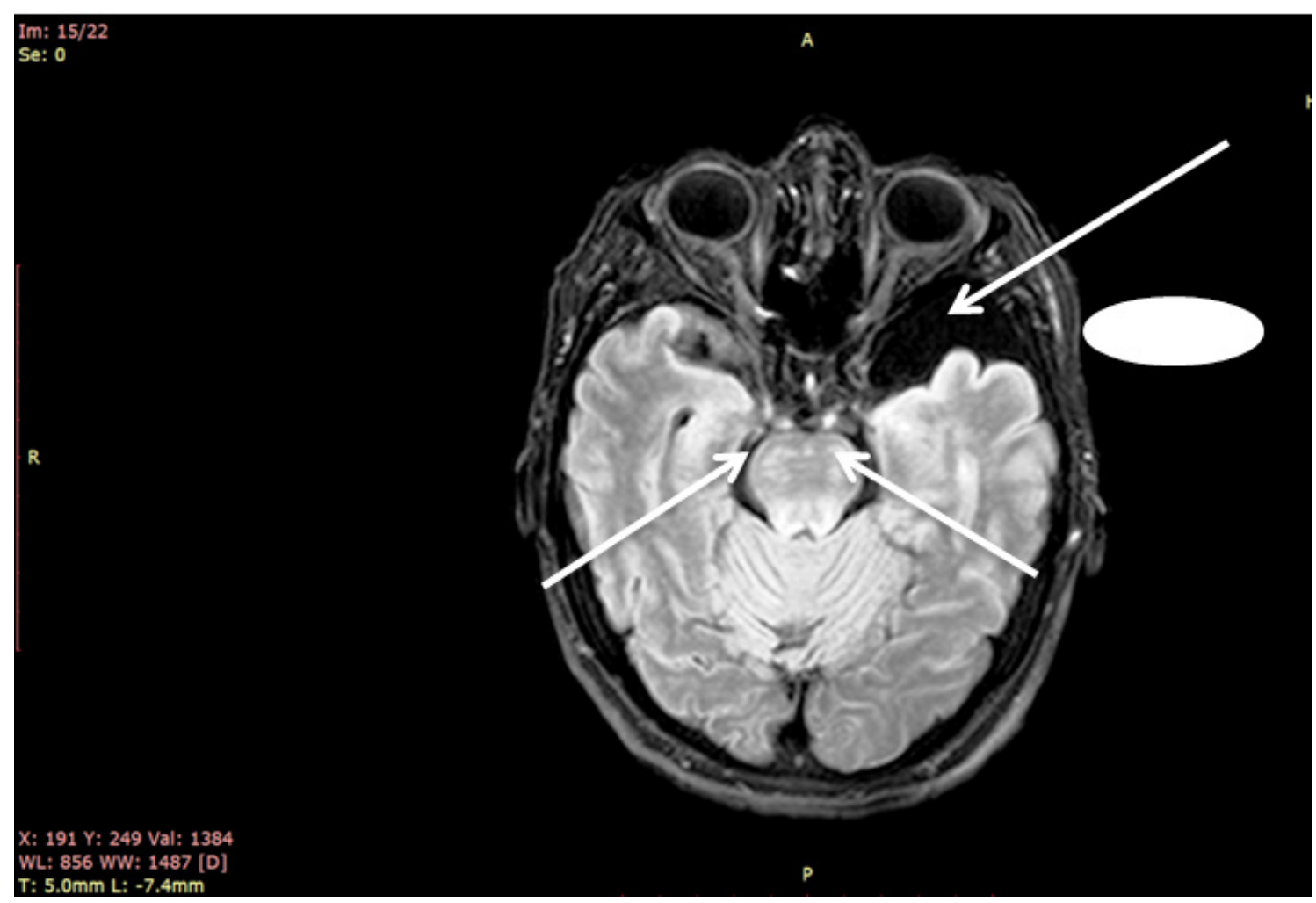

Figura 9. Señales de hiperintensidad en la protuberancia y quiste aracnoideo temporal.

y en el interés para realizar el test, minicognitivo de Folstein así como, a los resultados en cada uno de los ítems conocidos, en los que frente a las fallas, no le suscitaban incomodidad o ansiedad. La puntuación de la prueba neuropsicológica fue de 8 puntos con fallas en la orientación temporal, mencionando sólo el día de la semana, estaba desorientada en lugar y tuvo groseras fallas en la memoria inmediata retuvo dos de tres palabras y al minuto las olvidó completamente; nivel de atención igualmente afectada finalmente en la copia de los pentágonos entrelazados graficó un pentágono adjunto a un cuadrado. Presentó anhedonia frente a los resultados. No tuvo afasia, apraxia ni agnosia. Presentó incoordinación y adiadocinesia asimétrica, marcha atáxica zigzageante. Moderada debilidad en la fuerza muscular contra resistencia que se acompañó de reflejos vivos y ausencia de los signos de Babinski y el de Hoffmann. La exploración de la sensibilidad supeficial y profundas normales. Concluyéndose clínicamente el compromiso del vermis y los hemisferios cerebelosos, puso en evidencia el estado demencial al obtener una puntuación de ocho en el test de Folstein, no disartria, incoordinación axial y de los miembros y la marcha zigzageante, Cuadriparesia moderada en los miembros, sin signos patológicos, reflejos tendinosos presentes. Con estos resultados se produjo la exploración mediante resonancia magnética del cerebro (RM) contrastada, incidiendo en T1,T2 y el FLAIR, que mostró: atrofias comprometiendo las regiones fronto - témporo - parietales, el cuerpo calloso y el cerebelo ( figura 6 ). En la incidencia con el FLAIR se observó: hiperintensidad en las cápsulas internas, pedúnculos cerebrales y en las fibras transversas de la protuberancia. En forma accidental el hallazgo de un considerable quiste aracnoideo temporal izquierdo que no tuvo mayor implicancia clínica en el estado de la paciente (figuras 6, 7, 8 y 9)

\section{DISCUSIÓN}

La xantomatosis cerebrotendinosa (XCT) es un desorden autosómico recesivo de la síntesis de los ácidos biliares asociado con niveles anormalmente elevados de colastenol en el plasma con impregnación en los tejidos (9). Aunque considerada como un desorden raro, la XCT puede ser subdiagnosticada. Hay una marcada variabilidad en los síntomas y signos, tanto como en la severidad y la edad de inicio de los pacientes. Las manifestaciones clínicas que caracterizan a la XCT incluyen la diarrea crónica, cataratas bilaterales, xantomas tendinosos y disfunción neurológica, aún en etapas tempranas de la vida . El 
curso progresivo de la misma es debilitante o fatal, particularmente las manifestaciones neurológicas que pueden incluir, demencia y problemas neuropsiquiátricos. Los mecanismos por los cuales el colastenol se acumula en el sistema nervioso central no siempre bien comprendidos (10). Los precursores de los ácidos biliares entre los cuales se encuentra el ácido quenodeoxicólico ( AQDC ), atraviesa la barrera hematoencefálica, y eficientemente pueden convertirse a colestanol por neuronas, astrocitos, microglía y macrófagos derivados de monocitos humanos, conducirían al depósito del colastenol en el tejido cerebral. En nuestro caso, en quien tenemos escasos datos acerca de los antecedentes familiares , pudimos llegar al diagnóstico definitivo mediante la exploración clínica y el resultado de la biopsia de los tendones aquilianos, así como los cambos en las estructuras del encéfalo mediante la resonancia magnética. No pudimos determinar el colestanol plasmático, debido a no efectuarse en nuestro país. El estado demencial de nuestra paciente merece comentario aparte, pues no correspondería a las formas neuro-degenerativas, pues clínica y radiológicamente difiere de la enfermedad de Alzheimer y tampoco comparte características de la demencia vascular. En el aspecto genético, adicionalmente se han identificado 99 mutaciones diferentes implicada en la génesis de la XCT. No se ha establecido una correlación entre las mutaciones el cuadro clínico específico y la severidad del mismo. En fin no se ha determinado las variaciones fenotípicas en individuos de las mismas familias, así se han reportado, cuadros clínicos diferentes en las familias con la misma mutación genética, más aún, se ha reportado casos de gemelos quienes han presentado considerable fenotipo diferente (11). Esta enfermedad se ha descrito con mayor frecuencia entre italianos, japoneses, judíos sefardíes y en el norte de Africa. Los estudios realizados por Leisterdorf y colaboradores, entre judíos de origen marroquí y unos años más tardes por Lorinez entre americanos de origen europeo establecen una prevalencia aproximada de 3-5/100 000 habitantes (12).

En Chile existen dos publicaciones sobre xantomatosis cerebro tendinosa, una de ellas con diagnóstico clínico y de laboratorio, la segunda en un paciente con xantomatosis severa y en quien el estudio genético demostró la condición de heterocigoto para dos mutaciones diferentes en CYP27A1 causante de XCT $(7,8)$.

Los xantomas tendinosos aquilianos, biopsiados mostraron la naturaleza de la enfermedad, al igual que la presencia del arco senil tempranamente aparecidos, antes de los 30 años, las cataratas, la presencia de xantoma en el cuerno ventricular derecho por la especialidad y la osteoporosis estarían indicando un estado avanzado de la enfermedad por XCT. El estudio con resonancia magnética del cerebro (RM), puso en evidencia los cambios morfológicos del tejido cerebral, asi como, la penetrancia del colagenol en estructuras específicas empleando la incidencia del FLAIR.

Consideramos que la presente documentación del estado clínico de nuestra paciente corresponde a un estado avanzado de la enfermedad xantomatosa cerebro tendinosa ( XCT) con desarrollo de severa demencia asociada a ataxia cerebelosa. En la resonancia magnética del cerebro efectuada no se hallaron las hiperseñales en los núcleos dentados del cerebelo como suele aparecer en la mayor parte de las publicaciones. Este trabajo constituye el primer reporte efectuado en el Perú. Su carácter de autosómica recesiva obliga a estudiar a los familiares pues las formas asintomáticas se podrían beneficiar con el empleo del ácido quenodeoxicólico (AQDC), efectivo para la prevención de los depósitos del colastenol en los tejidos y frenar el desarrollo de la enfermedad (11). Consideramos con esta publicación alertar a la comunidad científica.

\section{CONCLUSIONES}

La presencia de los hallazgos oftalmológicos y las características morfológicas de las regiones aquilianas permitieron sospechar clínicamente de la la presencia de xantomatosis que se confirmó mediante los resultados histopatológicos de la biopsia efectuada. Los resultados de la exploración neurológica mostraron un estado demencial severo asociado a definidas alteraciones de la función cerebelosa.

El estudio mediante el empleo de la resonancia magnética cerebral fue decisivo para explicar las alteraciones anteriormente precisadas e interpretar la etiología al visualizarse la presencia del colastenol en diferentes localizaciones del tronco encefálico, así como, las regiones atróficas de los hemisferios cerebrales comprometidas y que explicaban la demencia como la ataxia.

De manera tal que se estableció el diagnóstico de xantomatosis cerebro tendinosa en la paciente en estadío avanzado de la enfermedad. 
Consideramos que esta comunicación constituye el primer reporte en nuestro país y esperamos contribuir con la comunidad científica en su conocimiento a efectos de facilitar la detección de la enfermedad en estadíos más tempranos.

\section{Correspondencia}

Marco A. Castañeda Reyna

Servicio de Neurología, Hospital Nacional Dos de Mayo

Jr. Moquegua 3655. San Martín de Porres, Lima,Perú Teléfono 51987185856

Correo electrónico: marcocastanedareyna@gmail. com

\section{REFERENCIAS BIBLIOGRÁFICAS}

1. Van Bogaert L, Scherer HJ, Epstein E. Une forme cerebrale de la cholesterinose generalised Paris: Masson et Cie; 1937.

2. Moghadasian MH. Cerebrotendinous xantomatosis : clinical course, genotypes and metabolic backgrounds. Clin Invest Med. 2004;27(1):42-50.

3. Björkhem I, Boberg KM, Leitersdorf E. Inborn errors in bile and biosynthesis and storage of sterols other than cholesterol. New York: McGrawHill;1995.p. 2073-99.

4. Dotti MT, Garuti R, Calandra S, Federico A. Clinical and genetic variability of CTX. Eur J Neurol. 996; 3 (Suppl 5): 12.

5. Dotti MT, Mondillo S, Plewnia K. Cerebrotendinous xanthomatosis : evidence of lipomatous hypertrophy of the atrial septum. J Neurol 1998;245: 723-26.
6. Dotti MT, Rufa A, Federico A. Cerebrotendinous xanthomatosis: Clinical phenotype with evidence of previously undescribed ophtalmologycal findings. J Inherety.Metab Dis. 2001; 24: 696-706.

7. Filippi J, Irarrázaval S, Peredo $\mathrm{P}$, Mellado $\mathrm{P}$. Xantomatosis cerebrotendinosa: Una causa de aumento del volumen bilateral del tendón de Aquiles. Rev Med Chil. 2009;137(6):815-20. do

8. Preiss Y, Santos J, Smalley S, Maiz A. Xantomatosis cerebrotendinosa: aspectos clínicos, fisiopatológicos, y genéticos. Rev Med Chile. 2014; 142 (5): 616-622.

9. Berginer VM, Salem J, Patel SB. Cerebrothendinous xanthomatosis. In: Rosemberg SN Pascual JM (editors). Rosenberg molecular and genetics basis of neurological and pshyquiatrics disease. London: Elsevier; 2015. pp. 589-598.

10. Bavner A, Shaafaati F, Hanson M, et al. On the mechanism of accumulation on the Cholestanol in the brain of mice with disruption of sterol27 hydrosylase. J Lipid Res. 2010; 51(9):2722-30. doi: 10.1194/jlr.M008326

11. Zadori D, Szpisjak L, Masdal L, et al. Different phenotypes in identical twins with cerebrotendinous xanthomatosis:case series. Neurol Sci. 2017; 38: 48183.

12. Pilo-de-la-Fuente B, Jiménez-Escrig A, Lorenzo JR, et al. Cerebrotendinous xanthomatosis in Spain: clinical, prognostic, and genetic survey. Eur J Neurol. 2011; 18(10):1203-11. doi: 10.1111/j.14681331.2011.03439.x

Recibido: 08/04/2019

Aceptado: 28/05/2019 